Original Article

\title{
POSSIBLE PROTECTIVE ROLE OF SODIUM SALICYLATE NANOEMULSION AND GINGER ON CISPLATIN-INDUCED HEPATOTOXICITY IN RATS (BIOCHEMICAL AND HISTOPATHOLOGICAL STUDY)
}

\author{
EL-SHEIKH S. E. ${ }^{*}$, EL-KHAYAT Z. ${ }^{2}$, HASSAN A. A. ${ }^{3}$, MOHAMED N. ${ }^{4}$, EL-NAGGAR M.5, OMARA E. ${ }^{6}$
}

*Egypt, Minoifia, Tala City, Bamb Village, Sadat Street Beside Elshiekh Zawiyatel, ${ }^{1}$ B. Sc. in Biochemistry 2020, ${ }^{2}$ Medical Biochemistry Department, National Research Centre, Dokki, Egypt, ${ }^{3}$ Chemistry Department, Minofia University, Egypt, ${ }^{2}$ Medical Biochemistry Department, National Research Centre, Dokki, Egypt, ${ }^{5}$ Pre-Treatment and Finishing of Cellulosic Fabric Department, National Research Centre, Dokki, Egypt, ${ }^{6}$ Department of Pathology, National Research Center, Cairo, Egypt

Email: safaamondy@yahoo.com

Received: 25 Jan 2020, Revised and Accepted: 23 Mar 2020

\begin{abstract}
Objective: To describe the preparation and characterization of nanoemulsion of sodium salicylate loaded butane tetracarboxylic acid (Bt-Sc-NPs). It also investigates the possible protective effects of Bt-Sc-NPs and \or medicinal plant ginger to evaluate the changes of liver functions, oxidative stress and histopathological investigations against cisplatin-induced hepatotoxicity.

Methods: Serum was used to determine alanine aminotransferase (ALT), aspartate aminotransferase (AST), $\delta$-glutamyl transferase ( $\delta$ GT), serum human laminin (LN) and tissue inhibitors of metalloproteinase1 (TIMP1). Liver tissue samples collected from the rats were used for the measurement of malondialdehyde (MDA), nitric oxide (NO) and paraoxonase 1 (PON1).

Results: The beneficial effects of Bt-Sc-NPs with its anti-inflammatory effect and the medicinal ginger with its antioxidant effect were observed. Injection of rats with cisplatin significantly increased serum ALT, AST, $\gamma$ GT, TIMP1 and LN. It also increased cisplatin-induced oxidative stress by a significant elevation in liver MDA, NO content; however, a significant decrease of PON1 content. While protection with Bt-Sc-NPs or ginger significantly improved these parameters. In addition, combination of both Bt-Sc-NPs and ginger significantly induced a decrease in serum ALT, AST, rGT, TIMP1 and LN. It also reduced cisplatin-induced oxidative stress by the significant reduction in liver MDA, NO content and elevation of PON1 content much more than protection with Bt-Sc-NPs or ginger alone.
\end{abstract}

Conclusion: Bt-Sc-NPs were synthesized using nanoemulsion with the help of homogenization and ultra-sonication waves. Combination with both of Bt-Sc-NPs and ginger showed a hepatoprotective role in ameliorating cisplatin-induced hepatotoxicity due to their anti-inflammatory and antioxidant effects.

Keywords: Hepatotoxicity, Liver, Oxidative stress, Silica nanoparticles, Cisplatin, Sodium salicylate, Ginger

(C) 2020 The Authors. Published by Innovare Academic Sciences Pvt Ltd. This is an open access article under the CC BY license (http://creativecommons.org/licenses/by/4.0/) DOI: http://dx.doi.org/10.22159/ijcpr.2020v12i3.38323 Journal homepage: https://innovareacademics.in/journals/index.php/ijcpr

\section{INTRODUCTION}

Adverse drug reactions are a major cause of patient morbidity and a significant cause of patient mortality [1]. Drug-induced liver injury may account for approximately $10 \%$ of all cases of acute hepatitis, $5 \%$ of all hospital admissions, and $50 \%$ of all acute liver failures. As well as it is a relatively common cause of acute liver disease and carries a mortality of around $10 \%$ [2]. Nowadays, liver damage becomes a very common cause of various metabolic disorders and can lead to even mortality [3]. One of the most important functions of the liver is the conversion of drugs into products that are more easily excreted [4]. Cisplatin (Cis-diamminedichloroplatinum II) which is one of the platinum-containing chemotherapeutic agents that are highly effective antineoplastic drug [5]. It is most commonly used in the treatment of tumors such as testicular, bladder, lung, stomach and ovarian cancers [6]. However, it causes many undesirable side effects on different tissues, including hepatotoxicity. Cisplatin-induced toxicity is most likely mediated by diverse oxidative damage to the cells [7]. The emergence of nanotechnology is likely to have a significant impact on the drugdelivery sector. Hence the main purpose of the nano delivery systems is to improve the natural biofate of potent drug molecules in the human body after their administration to significantly improve their efficacy and decrease their intrinsic adverse severe effects [8]. Among the antioxidants that have been tried against hepatoxicity are those that have been extracted from natural products (mainly medicinal plants and dietary components) [9]. Zingiber Officinale roscoe or ginger belonged to Zingiberaceae family, is a free radical scavenger and has been shown to be effective on injury in the experimental animals [10]. This article describes the preparation and characterization of nanoemulsion of sodium salicylate loaded butane tetracarboxylic acid (Bt-Sc-NPs) to reduce the side effects of sodium salicylate such as vomiting, nausea and gastric ulcers. In addition, our study investigates the hepatoprotective effect of the anti-inflammatory agent of (Bt-Sc-NPs) and the antioxidant agent (ginger) against cisplatin-induced hepatotoxicity in male Wistar albino rats in order to avoid falling in new drug toxicity against cisplatin-induced hepatotoxicity in rats.

\section{MATERIALS AND METHODS}

Chemicals

All reagents were used as received without further purification. Cetyltrimethylammonium bromide (CTAB, 99+ \%), tetraethyl orthosilicate (TEOS, 98), castor oil was purchased from Across Co (Germany). Ultrapure deionized (D. I.) water was generated using a Millipore Milli-Q plus system. Cisplatin and sodium salicylate were purchased from Sigma-Aldrich Chemical Company, St. Louis, MO, USA. Ginger extracts will be obtained from Arab Company for Pharmaceuticals and Medicinal Plants MEPACO, Cairo, Egypt.

\section{Animals}

Wister strain male albino rats weighing $180 \pm 20 \mathrm{~g}$, obtained from the Animal House, National Research Centre (NRC). The experiments were be carried out in accordance with guidelines and protocol approved by the Institutional Animal Ethics Committee. 
Preparation of sodium salicylate as a model drug-loaded butane tetra carboxylic acid

Sodium salicylate nanodrug was prepared using butane tetracarboxylic acid as a starting material by a direct method [11]. Firstly, the aqueous solution of sodium salicylate solution $(2 \mathrm{~mol} / \mathrm{l})$ was prepared in water contains $0.3 \mathrm{ml}$ of Tween 80 under magnetic stirring for $20 \mathrm{~min}$ at room temperature. Secondly, butane tetracarboxylic solution was prepared by dissolving $0.3 \mathrm{~g}$ of butane tetracarboxylic acid in $25 \mathrm{ml}$ of $\mathrm{H}_{2} \mathrm{O}$ and kept under magnetic stirring for $15 \mathrm{~min}$. After complete dissolution of butane tetracarboxylic, sodium salicylate solution coated with Tween 80 was added dropwise and kept under homogenization for $30 \mathrm{~min}$. The $\mathrm{pH}$ was adjusted to 8.0 using an aqueous solution of $\mathrm{NaOH}(1.0 \mathrm{~mol} / \mathrm{l})$. At this point, milky solution was observed, affirming the formation of emulsion which converted to nanoemulsion with the aid of homogenizer and ultra-sonication. Thus, the ultimate solution was submitted for homogenization for $30 \mathrm{~min}$ to enhance the dispersion of the synthesized nanoemulsion. Further technique such as ultra-sonication tool was used to improve the further dispersion for sodium salicylate nanoemulsion.

\section{In vitro experimental design}

Rats were randomly divided into eight equal groups, with ten rats in each group. Rats were given saline I. P (group 1) or carrier I. P 100 $\mathrm{mg} / \mathrm{kg} \mathrm{BW} /$ day (group 2) or cisplatin I. P. (20 mg/kg BW) (group 3). Some groups also received I. P injection of Bt-Sc-NPs $(100 \mathrm{mg} / \mathrm{kg}$ BW/day), according to Ganesan R. and Brian R, 2004 [12] either alone (group 4) or with cisplatin (group 5). Ginger was administered with stomach tube $310 \mathrm{mg} / \mathrm{kg} \mathrm{BW} /$ day alone according to Reaganshow et al., 2008 [13] (group 6) or with cisplatin (group 7) or both ginger and Bt-Sc-NPs (100 mg/kg BW/day) with cisplatin (group 8). The Bt-Sc-NPs, ginger or combination of both was administered for three weeks alone before the injection of cisplatin and then were continued for one week. Animals were then euthanized by decapitation. Blood and liver tissues were collected. Tissues were fixed in $10 \%$ neutral buffered formalin for histopathological examination or homogenized for estimation of liver parameters.

\section{Physical characterization of the synthesized nanoemulsion}

Visualization of morphology and structure of the as-prepared nanoemulsion based on sodium salicylate loaded tetracarboxylic butane acid was investigated using transmission electron microscopy (TEM). In order to carried out this analysis, a drop of nanoemulsion (negatively stained) was placed on copper grid and was allowed to dry in vacuum for $3 \mathrm{~h}$. Micrographs were acquired by using a transmission electron microscope (Tecnai-10, Philips) with a $\mathrm{W}$-source and operating at $80 \mathrm{kV}$. The surface morphology of the assynthesized butane tetracarboxylic acid with and without sodium salicylate was examined using scanning electron microscope (SEM; JSM-6360 LV; JEOL, Tokyo, Japan). The samples of nanoemulsions were suspended in water and vortexed for about $5 \mathrm{~min}$. The morphology of internal phase of emulsions was characterized by optical microscopy (CX41 RF, Olympus, Japan). Each sample was directly placed on the slide and visually observed through the digital eyepiece (AM423X, ANMO Electronic, Taiwan).

\section{Biochemical study}

Serum was used for the determination ALT, AST and $\delta \mathrm{GT}$, which were determined by using kit from Spectrum Company. Determination of serum LN and TIMP1 were performed by ELISA Kits, which were purchased from Bioneovan Company. The liver homogenate was used for estimation of lipid peroxidation by measuring the activity of MDA using the method of Ruiz-Larrea et al. 1994 [14], NO using Griess reagent, according to the method reported by Montgomery and Dymock, 1961 [15] and the arylesterase activity of paraoxonase using the method of Higashino et al. 1972 [16].

\section{Histopathological study}

The fixed samples were dehydrated in ascending series of ethanol, cleared in xylene, and embedded in paraffin wax. Sections $5 \mu \mathrm{m}$ thickness was prepared using a microtome stained with hematoxylin and eosin ( $\mathrm{H}$ and $\mathrm{E}$ ), and examination under a light microscope [17].

\section{Statistical analysis}

The collected data were coded, tabulated, and statistically analyzed using IBM SPSS statistics (Statistical Package for Social Sciences) software version 22.0, IBM Corp., Chicago, USA, 2013. Descriptive statistics were done for quantitative data as minimum and maximum of the range as well as mean $\pm \mathrm{SD}$ (standard deviation) for quantitative parametric data. ANOVA Test for more than two independent groups with post hoc Tukey HSD test.

\section{RESULTS}

The efficiency of the drugs was enhanced by encapsulation in suitable carrier. The choice of carrier mainly depended on its functional groups. Thus, tetracarboxylic butane acid was selected as a carrier due its capability to encapsulate the nominated model drug through the numerous functional groups (carboxylic groups). The utilization of Tween 80 was targeted to its ability to distribute the particles of sodium salicylate and kept them away from aggregation. Additionally, homogenization and ultra-sonication were utilized for enhancement the anti-agglomeration of the as prepared nanoemulsion. For confirmation the preparation of nanoemulsion, TEM, SEM, and optical microscope were used to clarify the surface morphology and particle shape of the formed sodium salicylate loaded butane tetracarboxylic acid. It was observed that, sodium salicylate loaded butane tetracarboxylic acid was successfully prepared in nanoform as observed in fig. 1 (A, B). The two images of TEM were taken at two different magnifications $(200 \mathrm{~nm}$ and $100 \mathrm{~nm}$ ) to clarify the size and shape of the formed nanoemulsion. It was observed that sodium salicylate loaded butane tetracarboxylic acid has very small size less than $50 \mathrm{~nm}$ which illustrate the prediction for the efficient of the formed nanoemulsion for medical purposes.
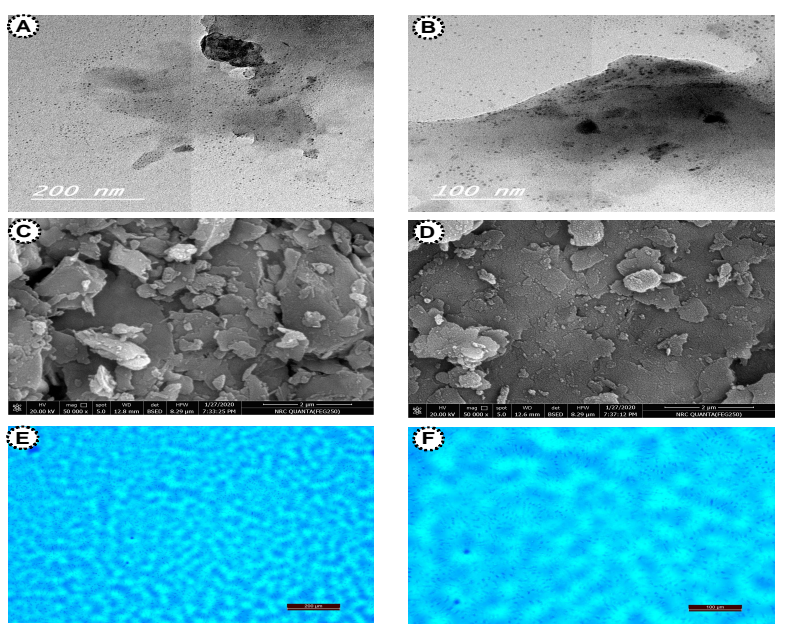

Fig. 1: (A, B) TEM, (C, D) SEM and (E, F) optical microscope of sodium salicylate loaded butane tetra carboxylic acid at different magnifications 
Moving to the surface morphology, SEM was utilized in order to examine the surface of butane tetracarboxylic acid nanoemulsion and sodium salicylate loaded butane tetracarboxylic acid. Fig. 1 (C) showed the morphological structure of butane tetracarboxylic acid nanoemulsion which exhibit agglomerate particles due to the absence of dispersion medium during examination. On the other hand, SEM image (fig. 1 D) displayed very small particles presented on the surface of butane tetracarboxylic acid which attribute to the existence of sodium salicylate. For further confirmation, optical microscope (fig. 1 E, F) was carried out to clarify the shape and size of sodium salicylate loaded butane tetracarboxylic acid at two different magnifications respectively. It could be clearly seen that, the particles of sodium salicylate loaded butane tetracarboxylic acid are formed with very small size and good distribution. The data obtained for sodium salicylate loaded butane tetracarboxylic acid from optical microscopy are in accordance with TEM and SEM data which revealed the successful preparation of nanoemulsion with well distribution and small size thanks to the presence of Tween 80 and dispersion tools; homogenizer and ultra-sonication.

\section{In vivo results}

Effects of cisplatin, Bt-Sc-NPs, ginger or their combination on the hepatic biomarker

Our results showed that no significant changes were observed in the mean value levels of serum AST, ALT, $\gamma \mathrm{GT}$ in carrier, Bt-Sc-NPs and ginger groups. However the induction of cisplatin significantly elevated $(p<0.001)$ these parameters compared to control group. In addition in the ginger protected group there were significant decrease $(p<0.05)$ in serum AST, ALT, $\gamma$ GT compared to cisplatin group. Besides the administration of Bt-Sc-NPs for $21 \mathrm{~d}$ before cisplatin significantly reduced $(\mathrm{p}<0.001)$ these hepatic alterations more than ginger can do. While the combination of both Bt-Sc-NPs and ginger highly significantly decrease $(p<0.001)$ this hepatic elevations much more than either Bt-Sc-NPs or ginger alone in comparison with cisplatin group (table 1 ).

Table 1: It shows serum AST, ALT and $\gamma$ GT of control and experimental rats

\begin{tabular}{llll}
\hline Groups & ALT (IU/l) & AST (IU/l) & rGT (IU/l) \\
\hline Control & $30.04 \pm 1.9$ & $77.9 \pm 8.7$ & $17.6 \pm 1.63$ \\
Carrier & $30.9 \pm 2.5$ & $86.2 \pm 8.19$ & $20.9 \pm 1.54$ \\
Cisplatin & $72.6 \pm 2.5 \mathrm{a}^{* *}$ & $125.9 \pm 1.4 \mathrm{a}^{* *}$ & $47.6 \pm 3.10 \mathrm{a}^{* *}$ \\
Bt-Sc-NPs & $29.2 \pm 2.48$ & $75.4 \pm 5.9$ & $18.7 \pm 1.72$ \\
Ginger & $31.6 \pm 3.2$ & $83.2 \pm 4.9$ & $20.5 \pm 1.56$ \\
Bt-Sc-NPs+cis & $54.9 \pm 2.4 \mathrm{c}^{* *}$ & $102.5 \pm 1.9 \mathrm{c}^{* *}$ & $30.5 \pm 2.48 \mathrm{c}^{* *}$ \\
Ging+cis & $70.9 \pm 1.5 \mathrm{c}^{*}$ & $112.8 \pm 1.9 \mathrm{c}^{*}$ & $40.6 \pm 0.87 \mathrm{c}^{*}$ \\
Ging+Bt-Sc-NPs+cis & $52.7 \pm 1.9 \mathrm{c}^{* *}$ & $99.4 \pm 3.4 \mathrm{c}^{* *}$ & \\
\hline
\end{tabular}

HG: Homogenous groups have the same letter, Number of cases= 8, Significant $\mathrm{P}^{* *}$ value $<0.001$, Significant $\mathrm{P} *$ value $<0.05$, $\mathrm{Pa}$ Significant difference compared to control group, Pc Significant difference compared to cisplatin group.

Effects of cisplatin, Bt-Sc-NPs, ginger or their combination on the oxidative stress and antioxidant biomarker

Our results showed that cisplatin significantly elevated $(\mathrm{p}<0.001)$ tissue MDA, NO levels compared to normal control group. It also exhibited a significant decrease in tissue PON1 level compared to the vehicle control group. Regarding groups protected with ginger, BtSc-NPs or their combination significantly restored the normal tissue levels of MDA, NO and increased the levels of PON1 indicating the antioxidant potential of these supplemented agents (table 2).

Table 2: It shows oxidative stress markers; Tissue MDA, NO and PONI as antioxidant of control and experimental rats

\begin{tabular}{llll}
\hline Groups & MDA $(\boldsymbol{\mu M} / \mathbf{l})$ & NO $(\boldsymbol{\mu M} / \mathbf{l})$ & PON1 $(\boldsymbol{\mu M} / \mathbf{l})$ \\
\hline Control & $97.4 \pm 3.5$ & $16.7 \pm 0.36$ & $17.3 \pm 0.49$ \\
Carrier & $110.8 \pm 2.1$ & $41.4 \pm 1.07 \mathrm{a}^{* *}$ & $92.3 \pm 4.4$ \\
Cisplatin & $241.3 \pm 5.8 \mathrm{a}^{* *}$ & $18.4 \pm 0.38$ & $50.2 \pm 4.3 \mathrm{a}^{* *}$ \\
Bt-Sc-NPs & $100.1 \pm 4.3$ & $17.8 \pm 0.61$ & $92.1 \pm 1.7$ \\
Ginger & $99.4 \pm 3.6$ & $30.2 \pm 2.6 \mathrm{c}^{* *}$ & $98.1 \pm 3.9$ \\
Bt-Sc-NPs+cis & $193.2 \pm 3.4 \mathrm{c}^{* *}$ & $34.9 \pm 1.8 \mathrm{c}^{*}$ & $66.2 \pm 3.4 \mathrm{c}^{* *}$ \\
Ging+cis & $204.1 \pm 1.6 \mathrm{c}^{*}$ & $26.2 \pm 1.0 \mathrm{c}^{* *}$ & $58.1 \pm 3.4 \mathrm{c}^{*}$ \\
Ging+Bt-Sc-NPs+cis & $178.8 \pm 2.9 \mathrm{c}^{* *}$ & $70.4 \pm 2.0 \mathrm{c}^{* *}$ \\
\hline
\end{tabular}

HG: Homogenous groups have the same letter, Number of cases $=8$, Significant $\mathrm{P}^{* *}$ value $<0.001$, Significant $\mathrm{P} *$ value $<0.05$, Pa Significant difference compared to control group, Pc Significant difference compared to cisplatin group.

Table 3: It shows Serum LN and TIMP1 of control and experimental rats

\begin{tabular}{lll}
\hline Groups & LN (ng/ml) & TIMP1 (ng/ml) \\
\hline Control & $66.8 \pm 2.1$ & $8.9 \pm 4.8$ \\
Carrier & $73.7 \pm 2.1$ & $14.4 \pm 5.5$ \\
Cisplatin & $219.9 \pm 8.4 \mathrm{a}^{* *}$ & $137.1 \pm 16.9 \mathrm{a}^{* *}$ \\
Bt-Sc-NPs & $70.9 \pm 3.5$ & $9.3 \pm 4.9$ \\
Ginger & $67.8 \pm 1.7$ & $10.3 \pm 5.4$ \\
Bt-Sc-NPs+cis & $192.7 \pm 2.8 \mathrm{c}^{* *}$ & $100.0 \pm 2.9 \mathrm{c}^{* *}$ \\
Ging+cis & $207.6 \pm 1.4 \mathrm{c}^{*}$ & $117.2 \pm 5.1 \mathrm{c}^{*}$ \\
Ging+Bt-Sc-NPs+cis & $194.7 \pm 1.7 \mathrm{c}^{* *}$ & $83.2 \pm 5.0 \mathrm{c}^{* *}$ \\
\hline
\end{tabular}

HG: Homogenous groups have the same letter, (Number of cases= 8), Significant $\mathrm{P}^{* *}$ value $<0.001$, Significant $\mathrm{P} * \mathrm{value}<0.05$, $\mathrm{Pa}$ Significant difference compared to control group, Pc Significant difference compared to cisplatin group. 
Effects of cisplatin, Bt-Sc-NPs, ginger or their combination on tissue inhibitor of metalloproteinase 1 and laminin

Cisplatin-induced hepatotoxicity was associated with increased serum LN and TIMP1 indicating the ability of cisplatin to solubilize the lipid components and alter the ion channels of bio-membranes. On the other hand protection with ginger, Bt-Sc-NPs or their combination significantly reduced them as compared to cisplatin group (table 3).

\section{Histopathological results}

Normal structure of the hepatocytes, central vein, sinusoidal spaces and prominent nucleus were observed in the liver section of control rats (fig. 2). Cisplatin exposure caused marked damage to hepatocytes in the form of degeneration, cytoplasmic vacuolation, centrilobular necrosis associated with mononuclear cells infiltration around central vein and the central vein was enlarged and congested. The sinusoid walls showed numerous Kupffer cells, hemorrhages with sinusoids dilatation, and nuclei are pyknotic with condensed chromatin (fig. 3).

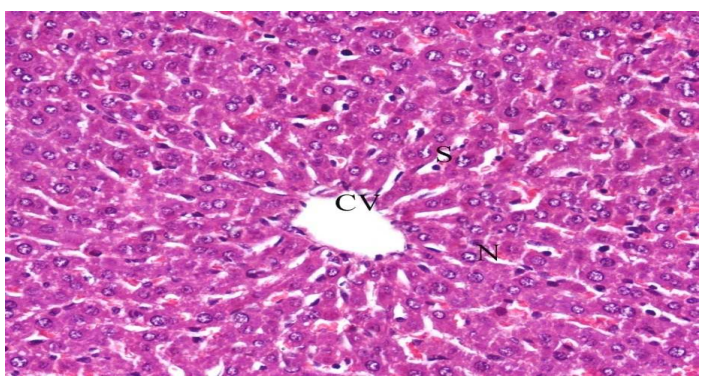

Fig. 2: It shows photomicrograph of section from liver of control group

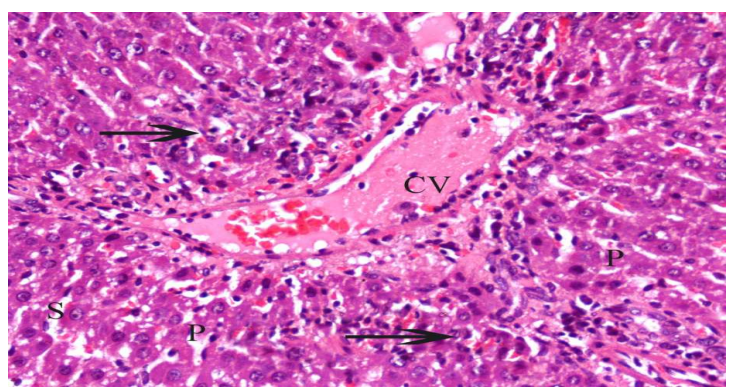

Fig. 3: It shows photomicrograph of section from liver of cisplatin ( $\mathrm{H}$ and $\mathrm{E} X \mathrm{400})$

In the group received ginger and carrier showed nearly normal structure with few Kupffer cells and hemorrhage blood sinusoids (fig. 4 and 5).

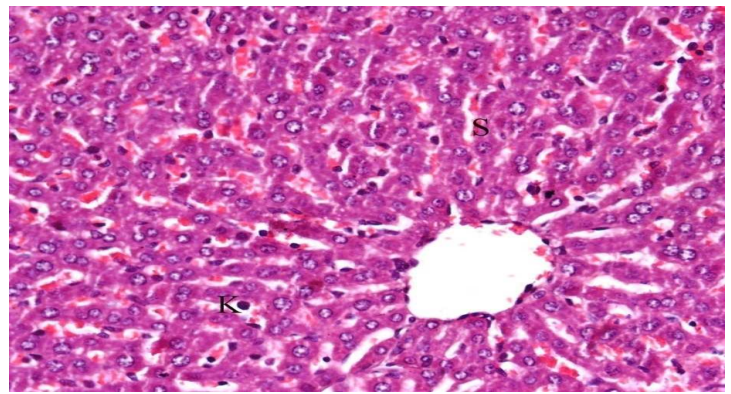

Fig. 4: It shows photomicrograph of section from liver of Carrier group (H and E X 400)

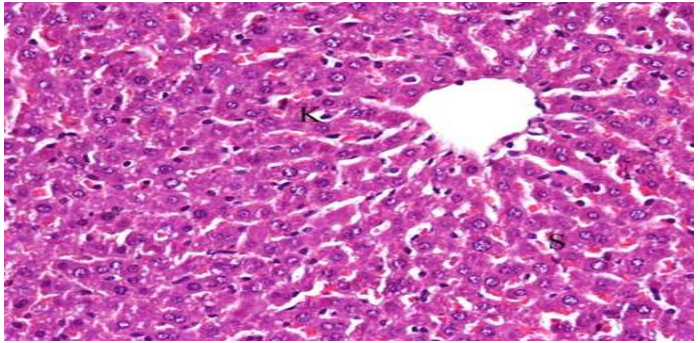

Fig. 5: It shows photomicrograph of section from liver of ginger group ( $H$ and $E X$ 400)

Histopathological examination of group received Sodium salicylate nanodrug showed almost the hepatic structure appear normal except few Kupffer cells and blood sinusoids (fig. 6). Hepatic sections of the rats that protected with Sodium salicylate and cisplatin showed ameliorate effect with inflammatory cells, necrotic cells, and congestion central vein and blood sinusoids (fig. 7).

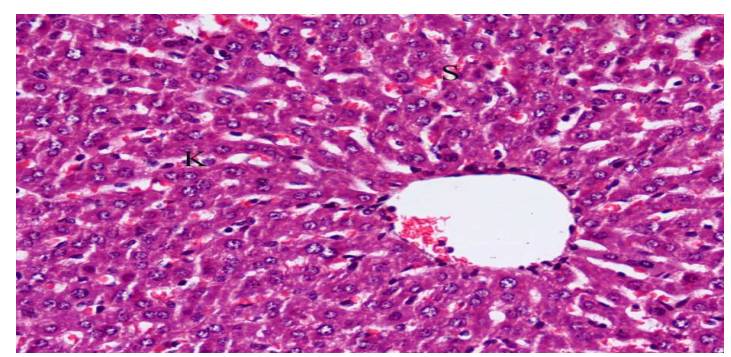

Fig. 6: It shows photomicrograph of section from liver of Sodium salicylate group (H and E X 400)

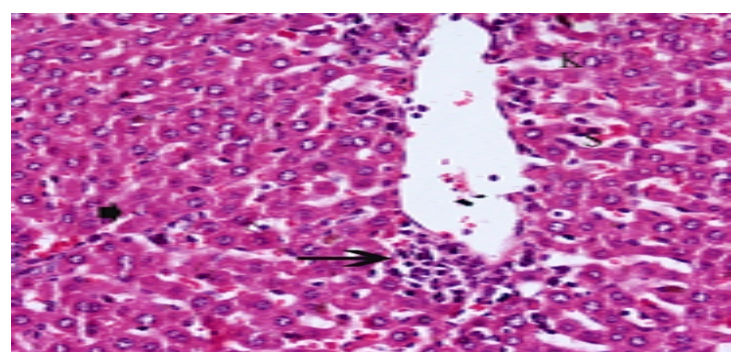

Fig. 7: It shows photomicrograph of section from liver of Sodium salicylate+Cis group ( $\mathrm{H}$ and $\mathrm{E} X \mathrm{400}$ )

Ginger+Cisplatintreatment attenuated the cisplatin induced liver tissue injury, with inflammatory cells, congestion central vein, blood sinusoids and pyknotic nuclei were observed (fig. 8). However, the pathological hepatic lesions induced by cisplatin were remarkably ameliorated by Ginger+Cisplatin+Sodium salicylate nanodrug except few inflammatory cells and congestion blood sinusoids (fig. 9).

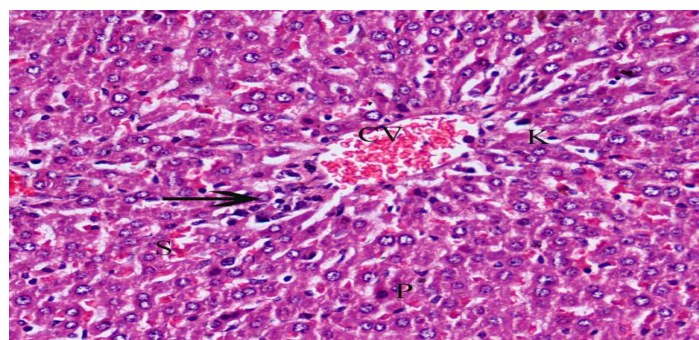

Fig. 8: It shows photomicrograph of section from liver of Ginger+Cisplatin ( $\mathrm{H}$ and $\mathrm{E} X \mathrm{400}$ ) 


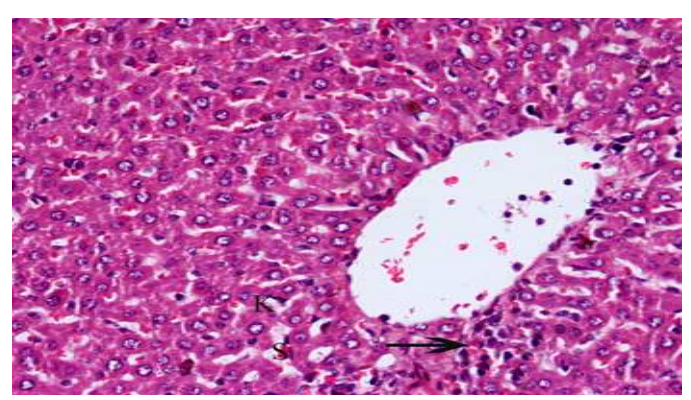

Fig. 9: It shows photomicrograph of section from liver of Ginger+Cisplatin+Sodium salicylate (H and E X 400)

\section{DISCUSSION}

Physicians prescribe drugs to prevent or treat disease. These drugs can be toxic to certain patients, however, because of genetic predisposition, nonselective action, or in appropriate use or administration of the drug [17]. Moreover, pharmaceutical and biotechnology companies spend years and millions of dollars in clinical trials to understand the safety and inherent toxicity of their drugs. Detoxification and metabolism of chemicals take place in the liver, making it the target organ for the toxicity [18]. Cisplatin is one of the most widely used cytotoxic therapeutic agents for the treatment of different cancers including testicular, bladder and lung cancers. It is an alkylating agent which at effective higher doses causes many adverse effects such as nephrotoxicity and hepatotoxicity [19]. Our results revealed that the used dose of cisplatin triggered severe liver toxicity as indicated by significant elevation in serum ALT, AST and rGT enzyme activities compared to control group due to their leakage from hepatocytes which could be a secondary event to cisplatin-induced liver damage. Another possible explanation of cisplatin-induced toxicity is that cisplatin induces a cascade of inflammatory reactions that play an important pathogenic role in cisplatin-induced tissue injury [20]. Our results confirm this previously reported by Niu et al. (2017) who reported that, cisplatin administration induced significant increase in serum ALT, AST and rGT enzyme activities due to liver cell damage [21]. On the other hand, Administration of Bt-Sc-NPs as protector for $21 \mathrm{~d}$ before cisplatin causes highly significantly decrease in the mean value levels of ALT, AST and rGT compared to cisplatin group which means that Bt-Sc-NPs has a main role in ameliorating inflammation of the hepatocyte. This could be due to the antiinflammatory effect of sodium salicylate drug which acts via inhibiting the production of prostaglandins from arachidonic acid by inhibiting COX activity. In addition protection with ginger significantly restores the changes of (ALT, AST, and $\gamma \mathrm{GT}$ ), due to components of ginger which could stabilize hepatocytes plasma membrane and prevent delivery of AST and ALTto the extracellular fluid leading to protecting membrane permeability indicating its hepatoprotective effect. Our results were in agreement with this of Haniadka et al. (2013) who investigated the hepatoprotective effects of ginger against liver toxicity due to its antioxidant effect [22]. In addition protection with Bt-Sc-NPs shows highly significant decrease in AST, ALT and $\gamma$ GT more than those results of protection with ginger which means that Bt-ScNPs is much more protective and safe than ginger can do. We also observe that administration with the combination Bt-Sc-NPs and ginger show highly significant decrease in AST, ALT and $\gamma \mathrm{GT}$, more than those of Bt-Sc-NPs or ginger alone which means that the liver is protected from damage produced by cisplatin. Those results were also supported by histopathological examination of the liver which showed that administration of cisplatin induced variousdegenerative changes in the liver tissue, including necrosis, hemorrhage, increased eosinophilic staining, pyknotic nuclei cells, congestion, and mononuclear cellinfiltration which confirmed the biochemical evidence of the oxidative stress. These results are in agreement with Alrashed et al. (2019) [4]. In contrast, protection with ginger and/or Bt-Sc-NPs before and after cisplatin obviously improved the histopathological changes induced by cisplatin. Oxidative stress is a common pathogenetic mechanism contributing to initiation and progression of hepatic damage in a variety of liver disorders. Cell damage occurs when there is an excess of reactive species derived from oxygen and nitrogen, or a defect of antioxidant molecules [23]. In our current study, Cisplatin administration elicited hepatic oxidative stress as demonstrated by a significant elevation of MDA as well as NOaccompanied by decrease in PON1 when compared to the control group in the liver tissue extracts. These results are in agreement with Omar et al. (2016) [20]. MDA, a product of lipid peroxidation, is specifically released as a result of the toxic effects of active oxygen radicals, which destroy unsaturated fatty acids in the cell membrane. Further, decomposition ofperoxidized lipids yields a wide variety of end products including MDA [24]. The elevation of MDA due to the increases in lipid peroxidation and oxidative stress is a reasonable explanation of liver injury induced by cisplatin in the following study. Moreover, many studies mentioned these increases in oxidative stress and ROS signalingcascade were associated with the activation of hepatic stellate cells (HSC's) which also produce ROS [25]. Cisplatin protected with Bt-Sc-NPs and/or ginger groups showed highly significant reduction in MDA and NO levels compared with cisplatintreated group. This might be due to ginger's ability to act as a free radical scavenger and antioxidant effect. Many studies showed that ginger was scavenging free radical by its potent antioxidant. Siddaraju andDharmesh (2007) reported that ginger-free phenolic and ginger hydrolyzed phenolic fractions exhibited free radical scavenging, inhibition of lipid peroxidation, DNA protection and reducing power abilities indicating strong antioxidant properties [26]. The results of the present work indicated that ginger hasprotective effect against hepatotoxicity induced by cisplatin. Also we observe that the administration with Bt-Sc-NPs highly significantly protected against the oxidative stress as indicated by lowering of MDA, NO when compared to cisplatin group which indicate the antioxidant effect of the Bt-Sc-NPs. Surprisingly, cisplatin pre-administrated with combined Bt-Sc-NPs and ginger group demonstrated highly significant reduced MDA and NO levels compared with cisplatin pre-administered with Bt-Sc-NPs or ginger which indicates that the liver was protected against oxidative stress and lipid peroxidation. It is proposed that NO is related to cisplatin-induced hepatotoxicity [27]. NO is a highly reactive molecule synthesized by liver parenchymal and nonparenchymal cells from L-arginine via the induction of the inducible form of nitric oxide synthase (iNOS) [28]. Furthermore, several researchers have determined that cisplatin treatment causes a significant increase in the level of NO in liver tissue. The increased tissue NOby cisplatin could be involved in the observed tissue damage via the formation of more reactive oxygen and nitrogen species by reacting with molecular oxygen [28, 29]. When present in high amounts, NO can react with the superoxide anion $\left(\mathrm{O}^{-}\right)$resulting in the highly reactive peroxynitrite (ONOO-) [30]. This highly toxic species reacts with lipids, proteins, GSH and DNA [31]. Our results are in agreement with this study. On the other hand, in this study, protection with Si-Sc-NPs or ginger associated with a highly significant decrease in liver NO compared to the group of cisplatin. PON1 is an enzyme synthesized in the liver and is found in plasma in association with high-density lipoproteins to prevent their oxidation. It is an important detoxifying enzyme and may play a preventative role against the development of different diseases [32] (Al-Helaly et al., 2014). Therefore PON1 should be defined as an antioxidant enzyme [21]. PON1 exertsits antioxidant and anti-inflammatory actions in the liver and is considered a biomarker of liver diseases [30]. PON1 is also sensitive to oxidative stress and decreased activity might thus reflect inactivation by reactive oxygen metabolites. Our results from this study also conceivable with that obtained by Al-Helaly et al., 2014, which indicated that PON1 was significantly decreased in liver in cisplatin group [32]. The decrease in PON1 activity observed in the current study after induction with cisplatin might thus reflects decreased synthesis by the intoxicated liver cells. However, the results from this article shows that there was significant increase in the levels of PONI in the ginger protected group which clarify the antioxidant properties of ginger in neutralizing or decreasing the free radicals, hence reducing oxidative stress and improving the condition of hepatocytes. In addition protection with Bt-Sc-NPs highly significantly increases PON1 which means that the sodium loaded butane tetra carboxylic acid nanoparticles is more protective and safe than ginger can do. We also observe that administration with the combination BtSc-NPs and ginger shows highly significant increase in the levels of PON1 much more than those of Bt-Sc-NPs or ginger alone which means that the liver is protected from damage produced by cisplatin 
indicating the protectiveeffect of both ginger and the sodium loaded butane tetra carboxylic acid nanoparticles model. In addition our present study revealed that TIMP-1 doesn't change in the control group. Besides in the group of Bt-Sc-NPs or ginger there were no significant changes in the mean value levels of TIMP1 compared to the control group which shows the idea that the HSCs were still in their quiescent state. However, those HSCs were activated by the existence of cisplatin leading to the highly production of extracellular matrix (ECM) andconsequently highly significant levels of the TIMP1. On the other hand, there was significant decrease in the mean value levels of serum TIMP1 in the double combination protected group (ginger and cisplatin) which means that protection with ginger prevented the high synthesis of ECM and decreased the level of serum TIMP-1 compared to cisplatin group. In addition protection with Bt-Sc-NPs was moreeffective than of ginger, demonstrating potential effect of this BtSc-NPs drug against hepatic damage as it isbelieved that activated HSCs and Kupffer cells are the major sources for TIMP-1 production during liver injury [33]. In our present study we also observe that the mean value levels of serum TIMP1 showed highly significant decrease in the combination protected group by both of ginger and Bt-Sc-NPs more than that of the protected group of Bt-Sc-NPs and/or ginger alone in comparison with cisplatin group which means that combination technique is much more efficient and safe. $\mathrm{LN}$ is one of the main glycoprotein of the basement membrane. This molecule takes part in a series of biological events such as regulation of the cytoskeleton. Sinusoidal cells and hepatocytes are able to synthesis of LN [34]. In our present study results, there was highly significant increase in the mean value level of serum LN in the cisplatin group compared to control group indicatethe ability of cisplatin to solubilize the lipid components and alter the ion channels of bio-membranes. While the protection with ginger exhibited significant therapeutic effect on reducing the levels of serum LN. In addition protection with $\mathrm{Bt}$-Sc-NPs causes highly significant effect in reducing the mean value levels of this marker indicating the effectiveness of Bt-Sc-NPs on hepatic regeneration after liver damage caused by cisplatin. The Increased production of LN in the liver and a lack of degradation of this glycoprotein by liver endothelial cells, are the main causes of elevated serum levels of this protein in cirrhotic and fibrotic liver. It is proposed that LN serum concentration is a susceptible screening test for hepatic fibrotic disease [34]. In our present study we also observe that the mean value levels of serum LN showed highly significant decrease in the combination protected group by both of ginger and BtSc-NPs more than that of the protected group of Bt-Sc-NPs and/or ginger alone in comparison with cisplatin group which means that combination protected group is much more efficient and enhances the liver damage caused by cisplatin. In our current study these results were confirmed by the histopathological analysis which showed that there were histopathological alterations in the liver tissue, including necrosis, hemorrhage, increased eosinophilic staining, pyknotic nuclei cells, congestion, and mononuclear cell infiltration in the group injected with cisplatin. Our results were in agreement with those of Alrashed et al. (2019) and Kart et al. (2010) [4, 35]. On the other hand histopathological examination of carrier, Bt-Sc-NPs and ginger groups showed that almost the hepatic structure appear normal except few Kupffer cells and blood sinusoids which confirm the safety and nontoxicity of these supplemented agents. However, protection with BtSc-NPs or ginger ameliorates histopathlogical damage with few inflammatory cells. In addition the pathological hepatic lesions induced by the cisplatin were remarkably ameliorated in the double combination protected group (Group 8) indicating the hepatoprotective, anti-inflammatory and antioxidant effects of Bt-ScNPs and ginger too. In the current study, administration with Bt-ScNPs, ginger or both ameliorate cisplatin induced hepatotoxicity at the biochemical and histological levels due to their protective, antioxidant and anti-inflammatory effects opening a new approach in diminishing drug induced hepatotoxicity.

\section{CONCLUSION}

The efficiency of the drugs was enhanced by encapsulation in suitable carrier. The choice of carrier was mainly depending on its functional groups. Thus, butane tetra carboxylic acid was selected as a carrier due its capability to encapsulate the nominated model drug through the numerous functional groups (carboxylic groups). The data obtained for Bt-Sc-NPs from optical microscopy are in accordance with
TEM and SEM data which revealed the successful preparation of nanoemulsion with well distribution and small size thanks to the presence of Tween 80 and dispersion tools; homogenizer and ultrasonication illustrating the prediction for the efficient of the formed nanoemulsion for medical purposes. In conclusion, Liu et al. (1998) reported that the drug accumulates in large quantities in hepatic tissue, especially when administered at high doses. This should be kept in mind before cisplatin dose planning because hepatotoxicity is another potential toxic effect of cisplatin even at nephrotoxic concentrations, which could be expected. The mechanisms involved in cisplatin-induced hepatotoxicity are complex and may include inflammation, oxidativestress injury and apoptosis [36]. In the current study, administration with sodium salicylate loaded butane tetracarboxylic acid (Bt-Sc-NPs), ginger or both ameliorate cisplatin induced hepatotoxicity at the biochemical and histological levels due to their protective, antioxidant and anti-inflammatory effects. In addition our findings show that Combination with both of Bt-Sc-NPs and ginger showed a hepatoprotective role in the amelioration of cisplatin-induced hepatotoxicity due to their anti-inflammatory and antioxidant effects by strengthening the antioxidant defense system through reducing reactive oxygen species and increasing antioxidant enzyme activities.

\section{FUNDING}

Nil

\section{AUTHORS CONTRIBUTIONS}

All the authors have contributed equally.

\section{CONFLICTS OF INTERESTS}

The authors declare no conflict of interests.

\section{REFERENCES}

1. Lazarou J, Pomeranz BH, Corey PN. Incidence of adverse drug reactions in hospitalized patients: a meta-analysis of prospective studies. JAMA 1998;279:1200-5.

2. Björnsson ES. Drug-induced liver injury: an overview over the most critical compounds. Arch Toxicol 2015;89:327-34.

3. Lahon K, Das S. Hepatoprotective activity of ocimum sanctum alcoholic leaf extract against paracetamol-induced liver damage in Albino rats. Pharmacogn Res 2011;3:13.

4. Alrashed AA, El-Kordy EA. Possible protective role of panax ginseng on cisplatin-induced hepatotoxicity in adult male albino rats (Biochemical and Histological Study). J Microscopy Ultrastructure 2019; 7:84.

5. Burger H, Loos WJ, Eechoute K, Verweij J, Mathijssen RH, Wiemer EA. Drug transporters of platinum-based anticancer agents and their clinical significance. Drug Resistance Updates 2011;14:22-34.

6. Tayem Y, Johnson TR, Mann BE, Green CJ, Motterlini R. Protection against cisplatin-induced nephrotoxicity by a carbon monoxide-releasing molecule. Am J Physiol Renal Physiol 2006;290:F789-F794.

7. Hussein A, Ahmed AA, Shouman SA, Sharawy S. Ameliorating effect of DL- $\alpha$-lipoic acid against cisplatin-induced nephrotoxicity and cardiotoxicity in experimental animals. Drug Discoveries Ther 2012;6:147-56.

8. Pathak Y, Thassu D. Eds. Drug delivery nanoparticles formulation and characterization. Vol. 191. CRC Press; 2016.

9. Heeba GH, Abd-Elghany MI. Effect of combined administration of ginger (Zingiberofficinale roscoe) and atorvastatin on the liver of rats. Phytomedicine 2010;17:1076-81.

10. Aryaeian N, Tavakkoli H. Ginger and its effects on inflammatory diseases. Adv Food Technol Nutr Sci Open J 2015;1:97-101.

11. Mohamed NA, El-Naggar ME. Amifostine-based nanoemulsion as promising protective agent for nephrotoxicity. J Innovations Pharm Biol Sci 2018;5:38-45.

12. Ramesh G, Reeves WB. Salicylate reduces cisplatin nephrotoxicity by inhibition of tumor necrosis factor- $\alpha$. Kidney Int 2004;65:490-8.

13. Reagan Shaw S, Nihal M, Ahmad N. Dose translation from animal to human studies revisited. FASEB J 2008;22:659-61.

14. Ruiz Larrea MB, Leal AM, Liza M, Lacort M, de Groot $H$. Antioxidant effects of estradiol and 2-hydroxyestradiol on iron- 
induced lipid peroxidation of rat liver microsomes. Steroids 1994;59:383-8.

15. Montgomery HA, Dymock JF. The determination of nitrite in water. Analyst 1961;86:414-6.

16. Higashino K, Takahashi Y, Yamamura Y. Release of phenyl acetate esterase from liver microsomes by carbon tetrachloride. Clin Chim Acta 1972;41:313-20.

17. Liebler DC, Guengerich FP. Elucidating mechanisms of druginduced toxicity. Nat Rev Drug Discovery 2005;4:410-20.

18. Watkins PB, Seeff LB. Drug-induced liver injury: summary of a single topic clinical research conference. Hepatology 2006;43:618-31.

19. Palipoch S, Punsawad C, Koomhin P, Suwannalert P. Hepatoprotective effect of curcumin and alpha-tocopherol against cisplatin-induced oxidative stress. BMC Complement Altern Med 2014;14:111-8.

20. Omar HA, Mohamed WR, Arafa ESA, Shehata BA, El Sherbiny $\mathrm{GA}, \mathrm{Arab} \mathrm{HH}$, et al. Hesperidin alleviates cisplatin-induced hepatotoxicity in rats without inhibiting its antitumor activity. Pharmacol Rep 2016;68:349-56.

21. Niu C, Ma M, Han X, Wang Z, Li H. Hyperin protects against cisplatin-induced liver injury in mice. Actacirurgicabrasileira 2017;32:633-40.

22. Haniadka R, Saxena A, Shivashankara AR, Fayad R, Palatty PL, Nazreth N, Baliga MS. Ginger protects the liver against the toxic effects of xenobiotic compounds: preclinical observations. J Nutr Food Sci 2013;3:1000226.

23. Medina J, Moreno Otero R. Pathophysiological basis for antioxidant therapy in chronic liver disease. Drugs 2005;65:2445-61.

24. Bentli R, Parlakpinar H, Polat A, Samdanci E, Sarihan ME, Sagir M. Molsidomine prevents cisplatin-induced hepatotoxicity. Arch Med Res 2013;44:521-8.

25. Siegmund SV, Brenner DA. Molecular pathogenesis of alcohol-induced hepatic fibrosis. Alcohol: Clin Exp Res 2005;29:102S-109S.
26. Siddaraju MN, Dharmesh SM. Inhibition of gastric $\mathrm{H}+$, $\mathrm{K}+$-ATPase and helicobacter pylori growth by phenolic antioxidants of Zingiberofficinale. Mol Nutr Food Res 2007;51:324-32.

27. Carnovale CE, Ronco MT. Role of nitric oxide in liver regeneration. Ann Hepatol 2015;11:636-47.

28. Kaltalioglu K, Coskun Cevher S. Potential of morin and hesperidin in the prevention of cisplatin-induced nephrotoxicity. Renal Failure 2016;38:1291-9.

29. Balabanli B, Coskun Cevher S. Alleviation of cisplatin-induced hepatotoxic damage: the synergistic effect of morin and hesperidin against oxidative stress. Res J Pharmacogn 2019;6:9-18.

30. Abdel Salam OM, Sleem AA, Youness ER, Mohammed NA, Omara EA. Bone marrow-derived protect against haloperidolinduced brain and liver damage in mice. Biomed Pharmacol J 2018;11:11-22.

31. Mansour HH, Hafez HF, Fahmy NM. Silymarin modulates cisplatin-induced oxidative stress and hepatotoxicity in rats. J Biochem Mol Biol 2006;39:656.

32. Al-Helaly LA. Studies on paraoxonase-1 isolated from amniotic fluid and its effect against cisplatin-induced hepatotoxicity and cardiotoxicity in rats. Rafidain J Sci 2018;27 (2E):42-56.

33. Jeong WI, Do SH, Jeong DH, Hong IH, Park JK, Ran KM, et al. Kinetics of MMP-1 and MMP-3 produced by mast cells and macrophages in liver fibrogenesis of rat. Anticancer Res 2006;26(5A):3517-26.

34. Rosa H, Parise ER. Is there a place for serum laminin determination in patients with liver disease and cancer? World J Gastroenterol 2008; 14:3628.

35. Kart A, Cigremis Y, Karaman M, Ozen H. Caffeic acid phenethyl ester (CAPE) ameliorates cisplatin-induced hepatotoxicity in rabbit. Exp Toxicol Pathol 2010;62:45-52.

36. Liu J, Liu Y, Habeebu SS, Klaassen CD. Metallothionein (MT)null mice are sensitive to cisplatin-induced hepatotoxicity. Toxicol Appl Pharmacol 1998;149:24-31. 